\title{
Methodology of Implementing Transformative Bioactive Hybrids in Built Environment to Achieve Sustainability
}

\author{
Yomna K. Abdallah \\ Universitat Internacional de Catalunya, iBAG (Institute for Biodigital Architecture \& Genetics) \\ | Barcelona, Spain | yomnaabdallah@uic.es
}

\author{
Alberto T. Estevez \\ Universitat Internacional de Catalunya, iBAG (Institute for Biodigital Architecture \& Genetics) \\ | Barcelona, Spain | estevez@uic.es
}

\begin{abstract}
Discrete responsive systems lack functional autonomous transformation, in response to environmental conditions and users' demands; due to shortage in direct integration of biological intelligence. Bioactive hybrids are sufficient solutions as they perform independente self-replication, differentiation of cellular structure, active metabolism, spatial propagation, adaptation, transformation, and morphogenesis. In this paper, a methodology is proposed for the design, fabrication and implementation of these hybrids in the built environment; highlighting their sustainability potentials, by merging synthetic biology, bioengineering and bioprinting, to achieve multiscale active responsiveness. The current work is part of research in biosynthesizing fibroblasts as transformative material in architectural sustainability.
\end{abstract}

Keywords: Transformative hybrids; Biodigital, Bioprinting; Robotic materials; Bioengineered systems.

\section{INTRODUCTION}

The key features of bioactive hybrids, are natural selfreplication and tissue differentiation. These features should be based on embedded biological intelligence in materials to multiply the hybrid cells for multiple purposes; such as locomotion for searching nutrients or escaping hazards, growth and self-healing, and physiological specification. Cellular differentiation through replication and transformation in the growth process is based on the natural biological intelligence sum of reactions of the surrounding environmental conditions, the internal physiological functions, the available resources, the genetic code and the validity of gene expression. synthesizing biological differentiation is the most challenging issue in bioactive hybrids, not just because of the complex physiochemical pathways involved in this process, but also because of its complex dynamic precision. Therefore, developing a transformative bioactive material application in architecture is an ambitious process that is still at laboratory scale. Modelling self-replication and tissue differentiation in bioactive hybrids depends on multidisciplinary research of bioengineering and synthetic biology. With the main tools of genetic manipulation and bio robotic materials. Bio robotic materials combine the complex systems of bioactive devices with bioactive materials. An example of attempts to embed biological intelligence of self-replication for self-healing and locomotion is exhibited in the recent work by S. Kriegman, et al., (2020) of designing and manufacturing reconfigurable organisms by aggregation of pluripotent blastula cells harvested from $X$. laevis embryos, that resulted in contractile tissue capable of self-locomotion in aqueous environment. (Kriegman, et al., 2020). Another example is the study conducted by S. Hamada, et al.,
(2019), where a dynamic DNA material with emergent locomotion behavior powered by artificial metabolism was developed resembling a slime mold by using an abstract design model similar to a mechanical system with dynamic properties, such as autonomous pattern generation and continuous polarized regeneration, enabled locomotion along the designated tracks against a constant flow. In order to synthesize bioactive materials and integrate them in architecture, a new design methodology must be adopted for attaining self-replication, differentiation and morphogenesis, that depends on synthesis of hybrids instead of assembly of discrete systems. For realizing such methodology, new digital fabrication techniques and tools are needed. These tools should support the biological features of continuous growth and translating genetic intelligence to be performed inherently and independently. since biological behaviors are singular and global in sync, they assist the mechanism of their morphogenesis and evolution being self-contained either at cellular level or at tissue level. These biological cultures are able of autonomous behavior. Designing a bioactive material that has these traits should provide to the maximum the survival of these materials by their own. Bioprinting is an emergent research field focused on printing bioactive materials that can support themselves for a certain period using an embedded source of nutrition. The current state of bioprinting lacks the durability of active growth in a longterm, as almost all printed bioactive materials need to be embedded in a host to grow. Another debatable aspect of printed bioactive materials is the limitation in tissue differentiation. In this paper the authors will identify the concept of bioactive hybrid materials highlighting their main potentials, limitations, possible fabrication methods and tools. In addition to proposing a methodology for employing these bioactive materials in achieving sustainability in the 
built environment through formal and functional transformation.

\section{METHODOLOGY}

\section{BIOACTIVE HYBRID MATERIALS}

A bioactive material is a biosynthesized material that contain bioactive agents, these agents are part or a whole of an organism; i.e., a whole cell of bacterium or a fragment of its DNA. Bioactive materials indulge the natural biological intelligence that is spontaneously inherited in the bioactive agents for performing certain ecological functions based on biological physiological processes preformed by these agents, for example, bioluminescence, biodegradation, biosensing of toxic compounds, bioelectrogenesis, etc.

A bioactive material is more complex than a biobased material, a biobased material might or might not include the living form of the bioactive agent, and its end result lack the ability of functional transition although it might have the ability to growth and formal development. A bioactive materialbincludes complex biological intelligence often based on biological networks for organizing multi scale responsive and adaptive behavior of the material enabling it of functional transition coupled with formal development in response to its environmental conditions (inner and outer environment). This functional transition coupled with the formal development that the bioactive materials have, yield a locomotive behavior that could be applied by various mechanisms; including a proliferative, a propagative mechanism or possessing special organs for motion, i.e. flagellates for gliding or swimming in motile environments. This locomotive behavior along with the functional transition are the main characteristics of an ideal bioactive hybrid.

Accomplishing functional transition indicates complex functional variety that are performed by the bioactive hybrid according to the compulsory environmental conditions, i.e. switching between enzymatic production to biosensing of certain chemical compounds, or switching from biosynthesis to bioluminescence activity. While achieving locomotive behavior includes synthesizing processes of proliferative cells or locomotive organs.

\section{MATERIALS AND METHODS}

\section{Synthesizing bioactive materials}

Synthesizing a full featured bioactive material hasn't exceeded lab scale until the moment, except for high profile applications in regenerative medicine or space excavations, due to the difficulty in combining synthetic biology practices with engineered biosystems including bio robotic materials and artificial/ biological intelligence, i.e. neural networks, nano robotics, etc. This difficulty arises from both the complexity in the hardware and software of the bioactive hybrid. The most challenging part is the nondetachable complexity of the intelligence behavior from the materialistic components as exhibited in Figure 1, as in the mass-energy equivalence paradox, where $E=M C^{2}$ (Bodanis, 2009), where the equivalent energy (E) can be calculated from the mass $(\mathrm{m})$ multiplied by the speed of light squared suggesting that even an everyday object at rest with a modest amount of mass has a very large amount of energy intrinsically. (Bodanis, 2009). Therefore, the bioactive hybrid synthesis practices must be by using an integrated method of case-based design. In the current study, the authors categorize definitive practices of bioactive hybrids synthesis as exhibited in Figure 2, in order to simplify the process for the community of architects and designers and encourage the adoption of such hybrids in the built environment. Synthesizing bioactive hybrids in this study is categorized into biosynthesis and bio robotic materials. The biosynthesis process depends on bioprinting techniques in fabricating the required bioactive material, while bio robotic materials depend on bioengineered systems.

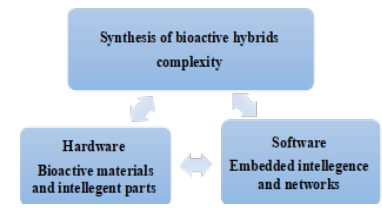

Figure 1: Schematic of the complexity of the synthesis of bioactive hybrids.

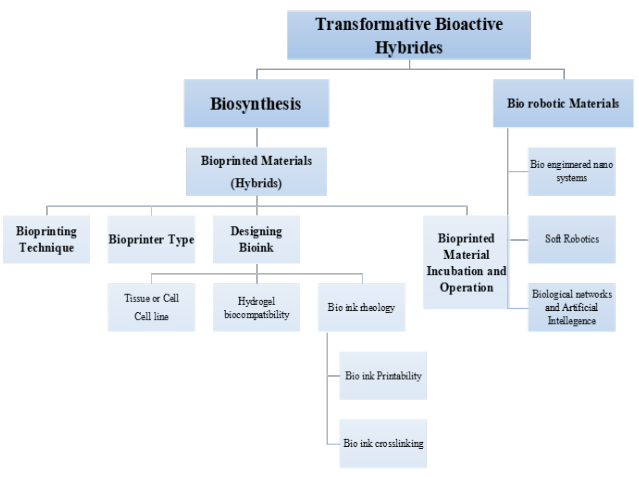

Figure 2: Schematic of integrated methods and tools for the synthesis of bioactive materials.

\subsection{Synthetic biology (SB)}

Synthetic biology combines genetic engineering, molecular biology, systems biology, biophysics, electrical engineering and evolutionary biology. Synthetic biology applies these disciplines to build biological systems for research, engineering, regenerative medicine, design and industrial applications. Synthetic biology is defined as "an emerging discipline that uses engineering principles to design and assemble biological components"(Wellhausen \& Oye, 2007), or biological modules, biological systems, and biological machines for useful purposes".(Nakano, 2013; Hayden, 2014; Robertson, et al., 2015). The goal of synthetic biology is to extend or modify the behavior of organisms to perform new tasks. However, SB is still a developing discipline that undergo extensive investigations about its drawbacks, for example; the inability to fully predict the functions of even simple devices in engineered cells and construct systems that perform multifaceted tasks with precision and reliability, due to several sources of uncertainty, some of which show the incompleteness of the available information about inherent cellular characteristics; such as the effects of gene expression noise, mutation, cell death, undefined and changing extracellular environments, and interactions with cellular context.The process of genetic engineering offers a broader definition of $S B$, associated with the practices of copying DNA sequences from the genome of an organism and, through recombinant DNA, importing the sequence from one organism to another, so that the host organism exhibits 
some characteristic of the donor (Robertson, et al., 2015). Recently it became possible to map entire genomes for individual organisms, which make it possible to make associations between specific DNA expression and the characteristics and behaviors of individual organisms. While DNA is described as the blueprint of life, the relationship between DNA sequences, the expression of proteins and the characteristics of biological systems are significantly more complex than this analogy suggests. While there are instances of isolated gene sequences resulting in clearly defined characteristics in an organism, much of what is understood in terms of the morphology and behavior of biological systems is derived from groups of different genes being expressed through the more complex proteome which is the entire population of proteins produced by a cell or organism, at particular growth stages or in particular environments. (Robertson, et al., 2015).

A highly prominent approach to SB is to abstract biological systems through design of more traditional forms of engineering. This emphasizes on simplifying the process of designing biological systems through the following,

- Engaging within an engineering design cycle, this includes a clear set of requirements, design, implementation, testing, verification and refinement, emphasizing on extensive simulation and modeling throughout the process.

- Describing DNA sequences and their products as standardized clusters, which are interchangeable and can be used to construct genetic circuits for different functions.

- Trading complex lab-based practices of recombinant DNA for using synthesized DNA (DNA, which has been coded, and 'printed out' from a computer). (Robertson, et al., 2015)

These computerized processes of conceptualizing SB as systems engineering indicates a wide dependence on bio informatics, that propose an invading tool to survey and compute an enormous amount of biological information; and also propose computation to simulate its' characteristics. However, this approach to biological systems' design is argued. Some suggest that complex biological networks cannot be reduced and partitioned into discrete clusters, this debate about the nature of biological processes stems from a long-standing argument between elementalism (studying systems through their reduction into parts) and organicism (the study of the system as an irreducible whole by recognizing the role of emergent complexity). In SB, an elementalist model emphasis DNA as the material of design manipulation. On contrary to the organicist view which the current work conforms that requires new designs to be considered from multiple possible perspectives and involve the manipulation of chemical, physical and cellular environments in concert with possible design outcomes which are emergent and difficult to describe with reference to the functional parts alone. (Robertson, et al., 2015).

There are three main techniques used in genetic engineering:the plasmid method, the vector method and the biolistic method. Plasmid Method, is used mainly for microorganisms such as bacteria, in this method, DNA molecules called plasmids are extracted from bacteria and broken down by restriction enzymes, as the enzymes break the molecules down, the plasmids develop a rough edge which is called the sticky end and is capable of reconnecting. These 'sticky' molecules are inserted into other bacteria where they will connect to the DNA rings with the altered genetic material. While, the vector method involves the transfer of a specific gene instead of a whole sequence. In the vector method, a specific gene from a DNA strand is isolated through restriction enzymes and is inserted into a vector. Once the vector accepts the genetic code, it is inserted into the host cell where the DNA will be transferred. The biolistic method is typically used to alter the genetic material of plants. This method embeds the desired DNA with a metallic particle such as gold or tungsten in a high-speed gun. Due to the high velocities and the vacuum generated during bombardment, the particle is able to penetrate the cell wall and inserts the new DNA into the cell.(www.sciencedirect.com).

Since architecture is a nonlinear interdisciplinary context that implies a bottom-up methodology when it comes to naturalizing its whole and parts, a recent approach that adopt this view presents the concept of material ecologies; according to Oxman, 2007; " the field operates at the intersection of biology, materials science, engineering, and computer science with emphasis on environmentally informed digital design and fabrication. With the advent of digital fabrication techniques and technologies, digital material representations have come to represent material ingredients, in other words, designers are now able to compute material properties and behavior built-in to formgeneration procedures." (www.materialecology.com).

A material model, which is based on observations of real biological systems, adopts the emergence of organization in living systems from constraints inherent in the materials themselves, and in their interaction with the environment. This approach in design paradigm, suggests a 'literal biological paradigm', suggesting that the designer should 'go beyond using shallow biological metaphors or a superficial biomorphic formal repertoire' and, through architectures of synthetic life, understand the built environment as 'a synthetic life-form embedded within dynamic and generative ecological relations'. (Hensel, 2006). Enabling a design process similar to cultivation than engineering. (Robertson, et al., 2015).

\subsection{Bioprinting}

Bioprinting is the utilization of $3 \mathrm{D}$ printing techniques to combine cells, growth factors, and bioactive materials. Bio active materials were originally used to fabricate biomedical parts that maximally imitate natural tissue characteristics. (Singh \& Thomas, 2015). Generally, 3D bioprinting utilizes the layer-by-layer method to deposit materials known as bioinks to create tissue-like structures. (Hinton, et al., 2015; Thomas, 2016).

Pre-bioprinting is the process of creating a printable model and choosing the materials that will be used. The first step is to obtain a sample (biopsy) of the organism. Common technologies used for pre-bioprinting are computed tomography (CT) and magnetic resonance imaging (MRI), to print with a layer-by-layer approach, the $2 \mathrm{D}$ images are sent to the printer to be printed. Once the image is created, certain cells are isolated and multiplied.(Shafiee \& Atala, 2016). These cells are mixed with a special liquefied material that provides oxygen and other nutrients to keep them alive. In some processes, the cells are encapsulated in cellular spheroids $500 \mu \mathrm{m}$ in diameter, this aggregation of cells does not need a scaffold, and are essential for placing in the tubular-like tissue fusion for processes such as extrusion.(Chua \& Yeong, 2016). In the second step, the liquid mixture of cells, matrix, and nutrients known as bioinks are placed in a printer cartridge and deposited using the scans. (Cooper-White, 2016). When a bioprinted pre-tissue is transferred to an incubator, this cell-based pre-tissue matures into a tissue. (Thomas,2016). Normally, $3 \mathrm{D}$ bioprinting for fabricating biological constructs includes dispensing cells onto a biocompatible scaffold using a successive layer-by-layer approach to generate tissue-like 
three-dimensional structures. Every tissue is naturally composed of different cell types, printing technologies vary in their ability to ensure stability and viability of the cells during the manufacturing process. Some of the methods that are used for 3D bioprinting of cells are photolithography, magnetic bioprinting, stereo lithography, and direct cell extrusion. (Chua \& Yeong, 2016). The primary benefit of 3D bioprinting lie in its capability of massproducing scaffold structures (Harmon, 2013). 3D bioprinting is based on three main approaches: Biomimicry, autonomous self-assembly and minitissue building blocks. (Hockaday, et al., 2012). In biomimicry approach, the main goal is to create fabricated structures that are identical to the natural structure involving both identical cellular and extracellular parts that are found in the tissues, which requires duplication of the shape, framework, and the micro environment of the tissues. (Yoo \& Atala, 2015). Therefore, it is necessary to understand the micro environment, the nature of the biological forces within it, the precise organization of functional and supporting cell types, solubility factors, and the composition of extracellular matrix. (Hockaday, et al., 2012). The autonomous self-assembly relies originally on the physical process of embryonic organ development as a model to replicate the tissues of interest. (Yoo \& Atala, 2015). When cells are in their early development, they create their own extracellular matrix building block, the proper cell signaling, and independent arrangement and patterning to provide the required biological functions and microarchitecture. A scaffold-free model that uses selfassembling spheroids subjects to fusion and cell arrangement to resemble evolving tissues. Autonomous self-assembly depends on the cell as the fundamental driver of hestogenesis, guiding the building blocks, structural and functional properties of these tissues. (Murphy \& Atala, 2014). while the minitissue approach of bioprinting is a combination of both the biomimicry and self-assembly approaches. (Thomas \& Singh, 2018). This approach relies on that organs and tissues are built from very small functional components, that are then manufactured and arranged into larger framework.

For realizing the bio printing process (Shafiee \& Atala, 2016). The bioink which is a material made from living cells that behaves much like a liquid, allow printing living tissue in desired shape. To make bioink, a slurry of cells is created that can be loaded into a cartridge and inserted into a specially designed printer, along with another cartridge containing a gel known as bio paper. There are three major types of printers; inkjet, laser-assisted, and extrusion printers that is used in this study to conduct experimentation on printing fibroplasts to creat fractal sustainable membranes. (Auger, et al., 2013). Extrusion printers print cells layer-by-layer for construction of a particular tissue to form a cell scaffold, just like 3D printing to create 3D constructs. (Ali , et al, 2017). This can be followed by the process of cell seeding, in which cells of interest are pipetted directly onto the scaffold structure. However, extrusion bioprinting allows printing of a cell suspension into a tissue construct with or without a scaffold support based on the process of integrating cells into the printable material itself, instead of performing seeding afterwards, this is called direct ink writing (DIW) which allows extrusion of high viscosity solutions, hydrogels, and colloidal suspensions. (Ali, et al., 2016; Shafiee \& Atala, 2016). Extrusion bioprinting is often coupled with UV light, which photopolymerizes the printed material to form a more stable, integrated construct. (Bajaj, et al., 2014). Materials for 3D bioprinting usually consist of alginate or fibrin polymers that have been integrated with cellular adhesion molecules, which support the physical attachment of cells. Such polymers are specifically designed to maintain structural stability and be receptive to cellular integration. The most common bioinks are cell-laden hydrogels, extracellular matrix (ECM)-based solutions, and cell suspensions. (Ji \& Guvendiren, 2017). Printing materials must be biocompatible, cyto-compatible, and bioactive, which indicates that the resulting 3D printed scaffolds should be physically and chemically appropriate for cell proliferation. Biodegradability is one more important factor, and ensures that the artificially formed structure can be broken down to be replaced by a completely natural cellular structure. Bio printing materials must be customizable and adaptable, being suited to wide array of cell types and structural conformations. (Augst, et al., 2006). Material printability is another pivotal parameter in the bioprinting process. Printability comprises two parts: the processability of the bioink formulation and the print fidelity associated with the mechanical strength of the printed construct to self-sustain a 3D structure post-printing. Printability involves solution viscosity, surface tension, and cross-linking properties. Viscosity is a crucial parameter for a bioink formulation as it affects both the print fidelity and cell encapsulation efficiency. High viscosity polymer solutions are less likely to flow easily so that the printed structure could hold its shape at longer times post-printing. However, they require higher pressures to flow. The bioink formulation is preferred to have a tunable viscosity to be compatible with different bioprinters. For instance, the viscosity of bioinks for extrusion-based DIW bioprinting ranges from 30 to $6 \times$ $107 \mathrm{mPa} \cdot \mathrm{s}$. For high viscosity bioinks used in extrusion bioprint, the shear-thinning characteristic is desired to compensate for the high shear stress associated with high viscosity. (Augst, et al., 2006). Alginates hydrogels are of the most used materials in tissue printing, as they are customizable, and can be fine-tuned to simulate certain mechanical and biological properties of natural tissue. Their ability to be tailored to specific needs allows them to be used as an adaptable scaffold material, which are suitable for a variety of tissue structures and physiological conditions. (Bajaj, et al., 2014). In the case of bioactive hybrids, it is useful to employ alginate as it has stability and slow degradation, which makes it persistent to environmental conditions until the full maturation of the bioactive hybrid cells.

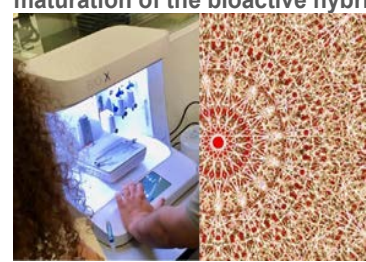

Figure. [3]. Extrusion bioprinter used in experimentation for printing the design of 3D fractal composition.

Alginate hydrogel that is suitable for extrusion printing is also often less structurally and mechanically sound; however, this issue can be mediated by the incorporation of other biopolymers, such as nano cellulose, to provide greater stability. The properties of the alginate or mixedpolymer bioink are tunable and can be altered for different applications and types of tissues. 


\subsection{Bio robotic materials}

Bio robotic materials comprise the ultimate integration between engineered biological systems such as; nano robots, genetic circuits, etc. and the bioactive materials that maintain the natural bioactive agents in their living form. Bio robotics combines the fields of biomedical engineering, bionics, cybernetics, and robotics to develop new technologies that integrate biology with mechanical systems to develop more efficient communication, alter genetic information, and create machines that imitate biological systems. (Axpe \& Oyen, 2016). Recently, many attempts have been done to incorporate bio robotics in architectural sustainability, one example of bio robotics applications in genetic circuits engineering is the MIT project of bacterial cells that produce biofilms incorporating gold nanoparticles and quantum dots to combine the advantages of living cells, which respond to their environment, produce complex biological molecules, and span multiple length scales, with the benefits of nonliving materials, which add functions of conducting electricity or emitting light. The MIT team used E. coli to produce biofilms that contain "curli fibers", which are amyloid proteins that help E. coli attach to surfaces, each curli fiber is made from a repeating chain of identical protein subunits called CsgA, that can be modified by adding protein fragments called peptides. These peptides can capture nonliving materials such as gold nanoparticles, incorporating them into the biofilms. (http://news.mit.edu/2014/engineers-design-living-

materials). By programming cells to produce different types of curli fibers under certain conditions, the researchers were able to control the biofilms' properties and create gold nanowires; and to communicate with each other and change the composition of the biofilm over time. The bio robotics applications in genetic circuits could be approached by computation of gene regulatory networks which prompted an analogy to the human-made electrical circuits, thus lead to the term 'genetic circuit.' This analogy can be made explicit by treating promoter/transcription-factor interactions (the building blocks of gene networks) as logic gates (the building blocks of computational electrical circuits). A discretization process can be applied to the continuously varying concentrations of transcription factors in a gene network. This abstraction results in a binary description of gene activity: at a given time-point, each gene is either 'on' expressing above threshold or 'off' expressing below threshold. Applying this notion to the concentration of a transcription factor provides, as an example, the comparison between repression of expression and a digital inverter. Promoters that are regulated by multiple transcription factors can be represented by multi-input logic gates. This implies a potential application in different ecological systems embedded in architecture, as for programming the real gene expression Boolean models to work as logic gates in specific functions; such as bio sensing for toxins. However, a crucial distinction between electrical circuits and gene circuits is how specific are the interconnections achieved, in an electrical circuit, all connections employ the same signal, while, the signal carriers for gene circuits (transcription factors) are mixed together in a single compartment, where unwanted interconnections are avoided through chemical specificity. This reliance on chemical specificity allows complex networks to operate on tiny spatial scales. (Schweitzer, 2007). An example of potential application of bio robotic hybrids in responsive architectural envelopes, is the BioHybrid Stingray Robot which is a swimming robot, created by researchers at Harvard University's Dept. of Bioengineering and Applied Sciences. The stingray robot is powered by rat muscle cells, with complex propulsion mechanism triggered by light, which allows the bio-bot to be steered around obstacles. The genetically encoded rat heart cells was engineered to respond to flashes of blue light, and aligned the cells along the ray's "fins" in a serpentine pattern. These muscles would allow the fins to flex downward, but to ensure they would return to the starting position, the team reverse-engineered a stingray's physiology to create a skeleton made of gold. The bio-bot was incubated into a saline solution filled with sugar to feed the rat cells and zapped with pulses of blue light. The rat muscles contracted sequentially along the serpentine pattern, causing a ripple effect mimicking the swimming motion of a live stingray, and it propelled the robot forward.

\section{(https://www.popsci.com/soft-robotic-stingray).}

As exhibited in the previous examples, architectural design could benefit profoundly from these advances in bio active hybrids, changing the methodological perspective in how to analyze and respond to architectural problems using biological sustainability intelligence by the direct act of the bioactive agents themselves. For realizing this, architectural form, and function need to be revolutionized to exit from the static rigidity constriction, to a dynamic ever-changing chaos that is mathematically accurate, functionally and ecologically sufficient in each time frame of its life cycle. For this aim, the authors develop a methodology for the application of bioactive hybrid materials in the built environment as follows.

\section{RESULTS AND DISCUSSION}

2. Methodology of implementing transformative bioactive hybrids in built environment

Integrating transformative bioactive hybrid materials in the built environment needs to be designed through a perspective of a life cycle or loop. A transformative bioactive hybrid is a dynamic system that is in continuous reaction with its inner and outer environment. It is an evergrowing system in form and function, that encompasses an ongoing exchange between energy and mass (material) in a complex response to functional requirements and environmental conditions. The dynamic feature presented in this study to control the bioactive hybrid transformation, responsiveness and morphogenesis infer the accurate design, simulation and manipulation of both the bioactive design and the recipient-built environment. This is to ensure the coherence in growth and evolution for bioactive hybrids and their surrounding environment as well. Figure. [4]. Describes a proposed lifecycle of the transformative bioactive hybrid in the built environment.

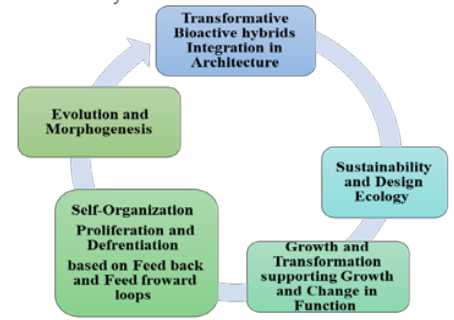

Figure. [4]. Bioactive hybrid life cycle for application in the built environment.

There are some ethical criteria to be considered when designing a bioactive hybrid material, this include; the validation of the bioactive hybrid that indicates long experimentation on these materials to monitor their functionality under different environments, conditions and 
even extremes, the directed bioactive intelligence, avoiding results of absolute intellegence integration such as, uncontrollablity of the hybrid's behaior or unpredictablity, that could hinder its functionality, therefore, certain functions should be specified and performed by genetic insertion on demand for them. Ethical criteria also include the life cycle design of the bioactive hybrid's in regard to ecologic system conservation in its main three phases: Emergence that includes the standard function of the hybrid and the initial growth phases in space, time and morphogenesis as well, with maintaining optimum conditions of safety measures. The second phase is Evolution, were a significant transformation in form, structure and functional capacities and qualities is performed by the bioactive hybrid, resulting in continuous growth and propagation in time and space, as the scale of performance would grow or decline. In this phase designers should consider growth margins and scenarios of form / function transformation respecting time frames and resources management; this would be conducted with the help of parametric simulation that utilizes mathematical biological models. The third phase is Decay or degradation, this includes two possible scenarios: the first is Finite decaying as the hybrid is biodegradable completely, and has no pollutants in any aspect, the second scenario is the Infinite evolution where the hybrid have an unlimited ability of fast and diverse adaptation physiologically in form and function. Yet this scenario is not achievable in the near future, as it demands high level of biological intelligence in the hybrid.

\subsection{Formal transformation in bioactive hybrids.}

This parameter consists of four regulators; materiality, structure, scale and evolution, however, each of these regulators never exist separately from the others. Structure and materiality are integrated and fused in the essence of transformative behavior of the bioactive hybrid, this indicates two options; either the structure and the envelop material are entirely integrated into one bioactive material to form a transformative selfsupporting monocoque structure, or that the structure is separated from the material but yet they act collaboratively and transform their form in sync. Attaining the first option is the more efficient solution in terms of formal coherency, functional sufficiency, and material sustainability. In order to synthesize this highly integrated material, a fractal dimension approach and an autonomous self-assembly building blocks bioprinting technique are used. The fractal dimension emerges from the self-similarity of the printed cells which are the building blocks of the bioactive material. The bioactive material inherited properties and transformative capacities lie in the genetically modified or biologically synthesized bioactive cells. Thus, the first step in producing a transformative bioactive material is bio engineering of the starting cell to perform specific ecological functions. The genetically modified cell also is optimized in terms of its physical properties, including its rigidity, viscosity, motility, stability under pressure and shear forces, and temperature resistance. In addition to chemical optimization for testing sensitivity to specific chemical compounds and toxins, solubility and $\mathrm{pH}$ sensitivity. All the previously mentioned constraints are in fact functional aspects that guaranty the durability and sustainability of the cell or the building block in the bio active material, however these functional constraints are the main controllers in the formal potentials of these materials.
Since fractal dimension and self-similarity are the main formal features of the bioactive material, scale plays a crucial rule in defining formal design iterations that could be organized from different configurations of the building blocks. Varying scale over the bioactive material fabric isn't realized by varying the scale of the building blocks (cells), but by varying the replication scale of the cells. This introduce the cell replication factor to identify the time consumed and the number of produced cells in one replication cycle. It depends on the liquid component of the bioink (hydrogel) to identify the interstitial distances between the replicated cells. However, the final spatial organization of the building blocks (cells) depend on post-bioprinting operation that include UV cross linking and material fastness. There are also number of constraints that control the final produced bioactive material composition and density; the cell viability factor is an important measure to count the persistent bioactive cell that survived from the main population of parent cells in the initial inoculum, similarly in each replication cycle, the cell viability factor can be estimated to measure the population of the active and alive

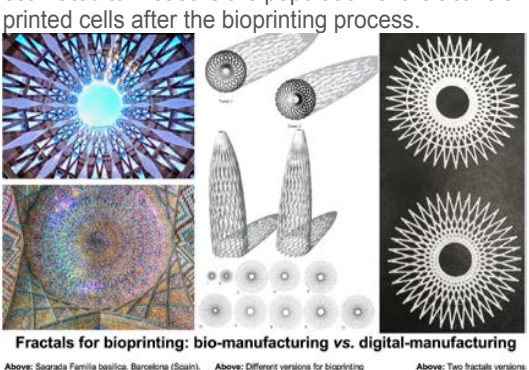

Figure. [5]. Fractal dimension in a continuous growth and transformation. Left, parametric architecture employing the conoid forms for formal compatibility to functional sufficiency (structural optimization and material sustainability). Right, comparison between the bioprinting compatibility in producing building blocks (fractals) that are able to evolve and transform according to its inner genotypes and outer phenotypes, and the digital printing in producing static form that lack the dynamic capacity to growth, transformation and evolution.

Formal transition is a key indicator of the bio active material dynamic development, since these materials inherited the natural capacity to replicate, this replication process including the fission's vector (direction axis), the cellular fusion and morphogenesis to different tissue controls the resulting texture and cellular composition of the bioactive material. Understanding formal properties of bioactive transformative materials is attained through dynamic simulation using mathematical models that resemble the natural cell properties and laws of replication, by using cellular simulation software. However, in order to predict the replication behavior of the bioactive material, computational tools are of secondary assessment, a laboratory experimentation that utilizes advanced microscopy and imaging systems that depict the cellular and intra cellular level, remains the main tool in testing these novel materials.

\subsection{Function: Proliferation and differentiation} based on Biological networks

The essential level of flexibility and transition between varied and different functions in bioactive hybrid materials requires a high level of biological complexity working on the genetic scale, to control the multilayered network of feedback loops with multiple stimuli, and requires a 
sufficient management of components, resources and materials.

This could be reached by employing Biological networks that constitute a type of communication technology. They receive information from the outside and inside of cells, integrate and interpret this information, and then activate a response. (Westerhoff, et al., 2017). One type of biological networks that is essential for functional customization in bioactive hybrids is Macromolecular networks, which confer intelligent characteristics, such as memory, anticipation, adaptation, reflection and network organization that reflect the type of intelligence required for the environments in which they were selected. (Westerhoff, et al., 2017). Another type of biological networks is Neural networks, they are mainly classified into two groups: the feedforward neural networks (FFNNs) where data is propagated from input to output using combinatorial machines, e.g., radial basis function (RBF), multilayer perceptron (MLP), and self-organizing map (SOM); and the recurrent neural networks (RNNs).

From feedforward neural networks several important loop motifs have been identified in both neuronal connectivity networks and transcriptional gene regulation networks. (Milo, et al., 2002), despite these networks operating on different spatial and temporal scales. The main motif to be applied in bioactive design is to reject transient input fluctuations/noises and activate output only if the input is persistent, a so-called persistence detector. (Alon, 2007). This is to avoid mal-functioning caused by over sensitivity to environment. In addition, a multi-input feedforward structure serves as a coincidence detector where the output is activated only if stimuli from two or more different inputs occur within a certain period of time. (Kashtan, et al., 2004). This motif is utilized for specific functional customization that requires more than one environmental condition shifting to occur in the bioactive hybrid response. While the recurrent neural networks RNNs have immediate biological application such as self-organizing dynamic systems and can describe complex non-linear dynamics, including both feedforward and feedback structures. This allows the network to reflect the input presented to it, but also its own internal activity at any given time.

Designing functional transition in bioactive hybrid also requires that the hybrid is able to learn and to remember various previous responses. Learning and memory are two important, counter posed features of intelligence. The former conforms new information, requiring flexibility in the network to produce complex dynamics, the latter retains old information, requiring stability in the network with sufficient storing capacity. Tradeoffs between the two can be modeled and observed using neural networks. In addition, feedback structures can increase network stability and exhibit near-perfect adaptation, where many properties of the system remain constant even when the system is subject to an environmental challenge or strong change in other network properties.( He, et al., 2013). Bioactive design functional transition needs decision-making to act and respond sufficiently and on time according to the imposed environmental challenges or internal requirements. For instance, decisions are made by monitoring the current state of the system, by processing this information and by taking action with the ability to take into account several factors such as recent history, the likely future conditions and the cost and benefit of making a particular decision. Bioactive agents are able to make decisions based on different criteria of information and to perform the decision-making using different mechanisms, utilizing different types of molecular networks. (Westerhoff, et al., 2017). This decision is made through the action of a complex hierarchical regulatory network, simultaneously involving gene expression, signal transduction, metabolic regulation and transport. (Van Heeswijk, et al., 2013). Another important feature of bioactive hybrid intelligence is the robust adaptation to changes in the environments. It describes an organism's response to an external perturbation by returning state variables to their original values before perturbation. Such robust adaptions include homeostasis (is the state of steady internal, physical, and chemical conditions maintained by living systems), as well as adaptive tracking of nutrient sources and evasion of harmful compounds. Relatively there are two types of robust adaptation intelligence in bioactive agents: the Long-term adaptations that often involve changes in genetic expression, such as gene mutations, transcription/translation activities or rewiring of gene regulatory networks, examples include adaptation to temperature, and the Short-term adaptation that typically involves regulation mediated either by Protein-protein interactions and covalent modifications in signal transduction pathways, or Direct substrate-product effects in metabolic networks. (Csete \& Doyle, 2002). Different regulation mechanisms in bioactive cells often occur at multiple levels simultaneously with a hierarchical structure. In this case, metabolic network regulation of a reaction rate can be achieved by the modulation of: Enzyme activity through a substrate, Enzyme covalent modification via signal transduction pathway, Enzyme concentration via gene expression. Such multi-level regulation corresponds to different control loops in the control system, which may ensure the robustness versus perturbations at various frequencies in the bioactive hybrid responding to changes in environmental conditions by functional customization. The metabolic regulation is identified as more of a proportional control action with limited range, (Samad, et al., 2002) and the gene expression regulation as more of an integral control action with potentially a wider range, but acting more slowly. Associative learning is another feature of bioactive hybrids. This type of learning specifies how several features in the environment, or within cells, change together. It suggests that the learner has a mechanism to encode mutual information. Associative learning in bioactive hybrids occurs when environmental variables are physically coupled. For example, the increase in the level of light intensity signals associated changes in the environment, such as increase in temperature. Bioactive hybrids weight these physical associations to better adjust their physiology in specific environments (Bonneau, et al., 2007), in some cases, even use the cues themselves to prepare or anticipate subsequent alterations to the environment, that time-scale for associative learning in bioactive agents (genetic regulations) is an evolutionary process and most likely involves genetic changes. (McGregor, et al., 2012; Carrera, et al., 2012). Typically, associative learning in biological populations involves some sort of social communication. Since bioactive hybrids do not respond to stimuli independently, but rather their internal networks direct common responses to diverse but related environmental signals, regulatory networks in bioactive hybrids can be reconstructed by measuring their response across a broad range of conditions. For instance, Gene regulatory networks, can be inferred in three simple steps: perturb cells across a broad range of relevant conditions, measure their transcriptional response in each environment, cluster similar gene expression patterns observed reproducibly across environments. Mining for genetic similarities among genes sharing a particular expression pattern, helps link these transcriptional modules to some of the molecular mechanisms responsible for regulating them. Self-awareness is a crucial 
aspect of functional customization in bioactive hybrids. Self-awareness can be described as the ability to recognize oneself as an individual separate from the environment and other individuals (singularity and global behavior indicator). Spatial propagation and orientation in bioactive hybrids are the result of functional sufficiency, formal development, transformation and evolution all together. The defining aspect of this criterion is the mechanism of propagation; the ability to move in all spatial direction in a natural wise. This includes the Motion mechanism where movement in the scale of micro, molecular and sub cellular level could be defined according to the resulting effect of growth, which is directed, by certain biological, biochemical and physiological processes inside the bioactive cell. This motion mechanism is categorized according to the resulting effect as: Macro, which affects the whole bioactive material by growth in the environment, or Micro, which affects certain tasks achieved by this movement inside the bioactive agent's building blocks (cells).

The Functional transition mechanisms according to this research scope, are micro movement of bioactive hybrid cells through liquids or over moist surfaces by using a variety of motility mechanisms (swimming, swarming, floating) and mostly use sensory mechanisms to control their movements. In order to make the decision of movement, the cell monitors the environment by means of multiple receptors in the cell membrane, the processing of this information inside the cell, is achieved by means of a signaling pathway. The level of the downstream protein of the signaling pathway, determines which movements the cell undertakes, using this mechanism, cells may perform wide array of motion patterns as Brownian motion or random walks, with the length of the periods of straight swimming dependent on the signal, resulting in movement toward or away from different stimuli. The other mechanism is the macro movement attained by proliferation and differentiation, this is based on physiological order, hestogenesis and tissue differentiation. Establishment of a growth site and the subsequent maintenance of the growth axis are the two strategies controlling this propagation. As a result, cell proliferation is subsequently confined to a discrete site, which ultimately leads to the formation of a new growth axis. The transport of growth supplies along with polarity establishment and maintenance, initiate the hybrid growth. Those supplies are delivered by chemical motors along the propagation axes of the extracellular matrix encompassing bioactive hybrid material. The regulation of these processes over time and space presumably accounts for much of the variation in the hybrid formal development and growth patterns.

2.3. Challenges and limitations in bioactive hybrid materials.

Applying bioactive hybrid materials in the built environment has many difficulties, aside from their syntheses process. There is no doubt that using such transformative materials in architecture balances to a great extent these difficulties, Bioactive hybrid materials are not solved yet for long term self-support applications, this means that they need to be augmented with nutrition and maintained in suitable environmental conditions to flourish and continue to grow and evolve espicially in case of bioactive hybrides based on animal cells. This limitation imposes multiple queries about the nutrition supply mechanism and the protection envelop that encompasses these materials. Another issue, is the formal composition resulting from the functional transformation each time the bioactive hybrid transforms. The adjustability of the bioactive hybrid to the physical spatial environment is a matter of learning and evolution that requires genetic mutation to be performed naturally by the bioactive hybrid's cells, this kind of learning is of long term adaptation that needs extensive experimentation on the long run to study and analyze the real behavior of these bioactive hybrids on act. The unpredictability of the bioactive hybrid behavior should be considered, as a bioactive hybrid is a complex biological being that involves inter reciprocal physiological pathways. This complexity of reactions and relations suggests the undetermined and unpredicted responsive behavior of the bioactive hybrid that controls the type of transformation required according to environmental conditions and users' demands.

\section{CONCLUSION}

Bioactive hybrid materials propose a multi-scale solution in attaining maximum functional customization coupled with formal transformation in response to inner and outer environmental conditions and users' demands to achieve design ecology. Transformation in bioactive hybrid materials is achieved by using synthetic biology to preform genetic modification to the used bioactive cells so that they perform certain ecological traits as part of their natural physiological pathways, such as bioluminescence or bioelectricity production alternately. Transformation in bioactive hybrids also employs bioengineered systems that utilizes bio robotics, artificial intelligence and complex biological networks to achieve multiscale responsiveness and decision making. Bioactive hybrid materials main characteristics are: the ability to proliferate and differentiate their tissue independently, self-sustain themselves in the built environment, perform responsiveness to environmental conditions through transformation, morphogenesis and evolution. However, these traits are still under extensive research due to the limitations that bioactive hybrid materials have. Bioactive hybrid material implementation in the built environment is limited by the unpredictability of their behavior through growth, propagation and transformation. Bioprinting is the main tool used for fabricating the bioactive hybrid materials. The process of bioprinting depend on many parameters to ensure the printability and efficiency of the post printed product, these parameters are categorized in three main aspects; the bioprinter, the bioink, and post printing processes of cross linking and material fastness. Implementing the bioactive hybrid materials in the built environment requires special criteria to suit the transformative feature of these materials. Design of bioactive hybrid's life cycle analyzes the hybrid different transitional phases with their functional customization and formal compatibility. The formal aspect of the proposed methodology for implementation of bioactive hybrid in the built environment, includes four main controllers; materiality, structure, scale and evolution. These formal controllers achieve a fractal dimension based on the utilized bioprinting technique of building blocks self-similarity. The functional aspect in the proposed methodology include the complex biological networks that bioactive hybrids utilize to organize the multi layered and multi scale information that they receive from their surrounding and inner environment to adjust their genotypes in response to these multiple stimuli followed by transformation in their phenotypes in the formal and functional aspect. These biological networks contain two main categories the are used in this study; the macromolecular networks and the neural networks. The functional aspect also includes the propagation and transition mechanisms used by bioactive hybrids to attain spatial propagation and motion in the built environment. 


\section{REFERENCES}

- Ali, Z., Kouzani, A. Z., Khoo, S. Y., Nasri-Nasrabadi, B. Kaynak, A. (2017). Development and analysis of a 3D printed hydrogel soft actuator, Sensors and Actuators A: Physical.

- Ali, Z., Kouzani, A. Z., Khoo, S. Y., Gibson, I., Kaynak, A. (2016). 3D printed hydrogel soft actuators, Region 10 Conference (TENCON), IEEE, 2272-2277.

- Alon, U. (2007). Network motifs: theory and experimental approaches, Nat. Rev. Genet, 8, 450-461.

- Auger, F. A., Gibot, L., Lacroix, D. (2013). The Pivotal Role of Vascularization in Tissue Engineering, Annual Review of Biomedical Engineering, 15, 177-200

- Augst, A. D., Kong Hyun, J., Mooney, D. J. (2006). Alginate Hydrogels as Biomaterials, Macromolecular Bioscience, 6(8), 623-33.

- Axpe, E., Oyen, M. L. (2016). Applications of Alginate-Based Bioinks in 3D Bioprinting, International Journal of Molecular Sciences, 17(12), 1976

- Bajaj, P., Schweller, R. M., Khademhosseini, A., West, J. L. Bashir, R. (2014). 3D Biofabrication Strategies for Tissue Engineering and Regenerative Medicine, Annual Review of Biomedical Engineering, 16, 247-76, 2014.

- Bodanis, D. (2009). E=mc^2: A Biography of the World's Most Famous Equation (illustrated ed.). Bloomsbury Publishing.

- Bonneau, R., Facciotti, M. T., Reiss, D. J., Schmid, A. K., Pan, M. Kaur, A. (2007). A predictive model for transcriptional control of physiology in a free-living cell, Cell, 131, 1354-1365.

- Carrera, J., Elena, S. F., Jaramillo, A., (2012). Computationa design of genomic transcriptional networks with adaptation to varying environments, Proc. Natl. Acad. Sci.

- Chua, C. K., Yeong, W. Y. (2016). Bioprinting: Principles and Applications, Singapore: World Scientific Publishing Co, 296.

- Cooper-White, M. (2016). How 3D Printing Could End The Deadly Shortage Of Donor Organs, Huffpost Science, TheHuffingtonPost.com, Inc.

- Cruz, B.M., Pike, S. (2008). Neoplasmatic design. Architecture Studies, 76, 6-7.

- Csete, M. E., Doyle, J. C. (2002). Reverse engineering of biological complexity, Science, 295, 1664-1669

- Hamada, S., Yancey, K. G., Pardo, Y., Gan, M., Vanatta, M., An, D., Hu, Y., Derrien, T. L., Ruiz, R., Liu, P., Sabin, J., Luo, D. (2019). Dynamic DNA material with emergent locomotion behavior powered by artificial metabolism, Science Robotics.

- Harmon, K. (2013). A sweet solution for replacing organs, Scientific American, 308(4), 54-55.

- Hayden, E.C. (2014). Synthetic-biology firms shift focus, Nature, 7485 (505), 598.

- He, F., Fromion, V., Westerhoff, H. V. (2013). (Im)Perfect robustness and adaptation of metabolic networks subject to metabolic and gene-expression regulation: marrying contro engineering with metabolic control analysis, BMC Syst. Biol, 7 131

- Hensel, M. (2006). (Synthetic) life architectures: ramifications and potentials of a literal biological paradigm for architectural design, Architecture Studies, 76, 18-25.

- Hinton, T. J., Jallerat, Q., Palchesko, R. N., Park, J. H. Grodzicki, M. S., Shue, H. J., Ramadan, M. H., Hudson, A. R., Feinberg, A. W. (2015). Three-dimensional printing of complex biological structures by freeform reversible embedding of suspended hydrogels, Science Advances, 1(9).

- Hockaday, L. A., Kang, K. H., Colangelo, N. W., Cheung, P. Y C., Duan, B., Malone, E., Wu, J., Girardi, L.N., Bonassar, L. J.,
Lipson, H., Chu, C. C., Butcher, J. T. (2012). Rapid 3D printing of anatomically accurate and mechanically.

- Ji, S., Guvendiren, M. (2017). Recent Advances in Bioink Design for 3D Bioprinting of Tissues and Organs-Front. Bioeng. Biotechnol.

- Kashtan, N., Itzkovitz, S., Milo, R., Alon, U. (2004). Topological generalizations of network motifs, Phys. Rev. E Stat. Nonlin. Soft Matter Phys, 70.

- Kriegman, S., Blackiston, D., Levin, M., Bongard, J. (2020). A scalable pipeline for designing reconfigurable organisms, PNAS, 117(4), 1853-1859.

- McGregor, S., Vasas, V., Husbands, P., Fernando, C. (2012) Evolution of associative learning in chemical networks, PLoS Comput. Biol.

- Milo, R., Shen-Orr, S., Itzkovitz, S., Kashtan, N., Chklovskii, D Alon, U. (2002). Network motifs: simple building blocks of complex networks, Science, 298, 824-827.

- Murphy, S., Atala, A. (2014). 3D bioprinting of tissues and organs, Nature Biotechnology, 32, 773-85

- Nakano, T. (2013). Molecular Communication, Cambridge.

- Robertson, M.D., Figueroa, C. R., Zhang, M. (2015). Materia ecologies for synthetic biology: Bio mineralization and the state space of design, Computer-Aided Design journal, 60, 28-39.

- Samad, H., Goff, J. P., Khammash, M. (2002). Calcium homeostasis and parturient hypocalcemia: an integra feedback perspective, J. Theor. Biol, 214, 17-29.

- Schweitzer, F. (2007). Active Brownian Particles: Artificia Agents in Physics, Stochastic Dynamics, 358-371.

- Shafiee, A., Atala, A. (2016). Printing Technologies for Medica Applications, Trends in Molecular Medicine, 22 (3), 254-265.

- Singh, D. Thomas, D. (2018). Advances in medical polymer technology towards the panacea of complex 3D tissue and organ manufacture, American Journal of Surgery.

- Thomas, D., Singh, D. (2018). Novel techniques of engineering 3D vasculature tissue for surgica procedures, The American Journal of Surgery.

- Thomas, D. J. (2016). Could 3D bioprinted tissues offer future hope for microtia treatment?, International Journal of Surgery, $32,43-44$

- Van Heeswijk, W. C., Westerhoff, H. V., Boogerd, F. C. (2013). Nitrogen assimilation in Escherichia coli: putting molecular data into a systems perspective, Microbiol. Mol. Biol. Rev, 77, 628-695.

- Wellhausen, R., Oye, K. A. (2007). Intellectual Property and the Commons in Synthetic Biology: Strategies to Facilitate an Emerging Te, W97 binnenwerk-8, Rathenau Constructing Life, IEEE, Atlanta Conference on Science, Technology and Innovation Policy.

- Westerhoff, H. V., Brooks, A. N., Simeonidis, E., GarcíaContreras, R., He, F., Boogerd, F. C., Jackson, V. J. Goncharuk, V., Kolodkin, A. (2014). Macromolecular networks and intelligence in microorganisms, Front Microbiol, 5, 379.

- Yoo, J., Atala, A. (2015). Bioprinting: 3D printing comes to life, Manufacturing Engineering.

- "Biolistic Transformation- an overview, ScienceDirect Topics". www.sciencedirect.com. Retrieved 2020-04-03.

- http://www.materialecology.com- http://2012.acadia.org.

- heterogeneous aortic valve hydrogel scaffolds, Biofabrication, 4(3)

- http://news.mit.edu/2014/engineers-design-living-materials$1 / 30 / 2018$

- https://www.popsci.com/soft-robotic-stingray 